\title{
Investigation of the effect of ultrasonic surface treatment before ion implantation on the structure of the surface layer of aluminum alloy 1201
}

\author{
Viktor Ovchinnikov ${ }^{1 *}$, Irina Kurbatova $^{1}$, and Nadezda Uchevatkina $^{1}$ \\ ${ }^{1}$ Moscow Polytechnic University, B. Semenovskaya st., 38 Moscow, 107023, Russian Federation
}

\begin{abstract}
The aim of this study was to study the properties of 1201 aluminum alloy after ultrasonic treatment and their evolution during subsequent ion implantation using the monotectic $\mathrm{Cu}-\mathrm{Pb}$ alloy as the cathode material of the implant. It is shown that during ultrasonic treatment the surface layer of aluminum alloy 1201 undergoes significant changes. A nanocrystalline structure with a grain size of less than 200 microns is formed in it. Ultrasonic treatment of the surface of the target from alloy 1201 before implantation leads to a decrease in the depth of penetration of ions to $160-180 \mathrm{~nm}$ and the appearance of amorphization in the implanted layer.
\end{abstract}

\section{Introduction}

Significant progress in the development of the fundamental foundations of ion implantation in recent decades has been achieved thanks to intensive research in the field of modification of the properties of metallic materials. The study and generalization of the laws of physical processes caused by ion bombardment in a surface doped layer is an urgent task [1-3].

During ion implantation, the level of properties of the surface layer obtained as a result of irradiation depends on the mode and types of introduced ions, but also on the initial state and level of properties of the target surface before implantation. One of the areas of research is ion implantation after ultrasonic treatment.

Ultrasonic processing (RCD) is an advanced technology for finishing and hardening of metals by pressure, allowing you to replace the classical static methods of PPD according to the schemes of rolling and sliding - rolling and smoothing [4-7].

The deformation of the surface layer is performed by an acoustic head oscillating with an ultrasonic frequency, into which a carbide indenter is soldered. Due to the influence of ultrasonic vibrations, the resistance of the metal to plastic deformation is significantly reduced. At the final stage of the manufacturing process of the part, as a rule, various finishing methods are used for technological support of the quality of the surface layer -

\footnotetext{
Corresponding author: uchevatkina@yandex.ru
} 
abrasive finish, heat quenching, coating, surface alloying and plastic deformation. To achieve the maximum hardening effect, combined processing methods are used [8].

When solving the problem of increasing the wear resistance of parts made of titanium alloys, it is important to bear in mind that the wear resistance of the finished parts will be determined not only by the conditions and modes of implantation, but also by the type of surface treatment before implantation.

The aim of the study was to assess the effect of ion implantation using alloy implant cathodes based on immiscible components on the structure of an aluminum alloy.

\section{Experimental details}

As a material for the study, aluminum alloy plates with a thickness of $5 \mathrm{~mm}$ and dimensions 100x200 mm were used.

Ultrasonic processing was carried out using an IL10-0.63 generator (Russia), connected to an oscillating system consisting of a magnetostrictive transducer, a waveguide hub, and an acoustic head with a welded carbide indenter.

Ion implantation of alloy samples was carried out in an ion implantation unit equipped with two independent ion sources: a metal ion source and a gas ion source (the so-called double-beam installation).

The source of metal ions refers to the type of sources with a vacuum arc in metal vapors and represents a new type of source, where a vacuum arc in metal vapors is used as the plasma medium from which ions are extracted. The source operates in a pulsed mode and generates pulsed beams of metal ions.

A distinctive feature of this type of ion sources is that they form a polyenergy ion beam, i.e. the beam composition includes not only singly charged, but also multiply charged ions.

During operation, the source of metal ions provides a wide-aperture beam of circular cross section with a diameter of $200 \mathrm{~mm}$ at an ion beam current of $0.1 \ldots 1 \mathrm{~A}$. The accelerating voltage is $40 \mathrm{kV}$. In this source, the arc burns with pulses with a frequency of $50 \mathrm{~Hz}$ and a pulse duration of $\sim 300 \mu \mathrm{s}$. The implantation dose of the studied samples was $10^{17}$ ion $/ \mathrm{cm}^{2}$. A monotectic $\mathrm{Cu}-\mathrm{Pb}$ alloy with a lead content of $36 \%$ was used as the cathode material.

To study the effect of processing on the microhardness of the surface of 1201 aluminum alloy, the Affri 251 VRSD universal digital hardness tester (Italy) was used. Determination of the level of residual stresses in the samples after ultrasonic treatment (RCD) was carried out on a general-purpose automatic multifunctional X-ray diffractometer DRON-7 (Russia).

Determining the nature of the microstructure and the distribution of dislocations in the treated surface layer of the samples, a JE E L JEM-2100 transmission electron microscope (Japan) was used.

Ultrasonic processing refers to dynamic PPD methods. It is a process of impulse penetration, oscillating with the ultrasonic frequency of the indenter in the surface layer of the workpiece, and causes plastic flow of the metal in the contact zone. The generator, powered by a single-phase AC network with a frequency of $50 \mathrm{~Hz}$ and a voltage of $220 \mathrm{~V}$, generates oscillations of an electromagnetic field with a frequency of $20 \mathrm{kHz}$, which are transformed by means of an electro-acoustic transducer into mechanical vibrations of a waveguide-concentrator and, after increasing the amplitude, are transmitted to a working body - an acoustic head with a soldered carbide indenter [8].

The ultrasonic treatment mode (frequency and amplitude of vibrations, shape and radius of the indenter, speed of the main motion and feed rate, static force) determines the quality parameters of the surface layer (roughness, waviness, microhardness, residual stresses, hardening depth, dislocation density) that affect operational properties of machine parts 
(strength, wear resistance, contact stiffness, fatigue resistance, corrosion resistance, tightness of joints, friction coefficient, etc.) [9-12].

As the basic mode of the RCD, a mode with a static force value of $150 \mathrm{~N}$ and a feed rate of $180 \mathrm{~mm} / \mathrm{min}$ was selected according to the results of the work.

\section{Results and discussion}

Samples of the aluminum - copper 1201 alloy (aluminum content 3.8 at.\%) were subjected to intense ultrasonic irradiation in an aqueous medium using a VIP 1000hd ultrasound generator (Heilscher, Germany) operating at a frequency of $20 \mathrm{MHz}$. The calculated radiation power was $57 \mathrm{~W} / \mathrm{cm}^{2}$. The surface microrelief of the irradiated samples was studied using a LE $\cdot-1420$ scanning electron microscope with an $\mathrm{R} \cdot$ ntec Xray microanalyzer. Corrosion studies were performed by electrochemical impedance spectroscopy using a Gamry FAS2 femtostat in the frequency range $5 \times 104-10-3 \mathrm{~Hz}$.

As it can be seen from the figure 1 electron-microscopic images of the surface of the samples of aluminum-copper alloy, the effect of intense ultrasound leads to a change in the morphology of the surface, which acquires a scaly-like structure. Studies by X-ray fluorescence spectroscopy showed that the result of ultrasonic exposure is surface oxidation, and when the radiation power is used, the amount of surface oxide formed is saturated after the first 10 minutes of exposure.

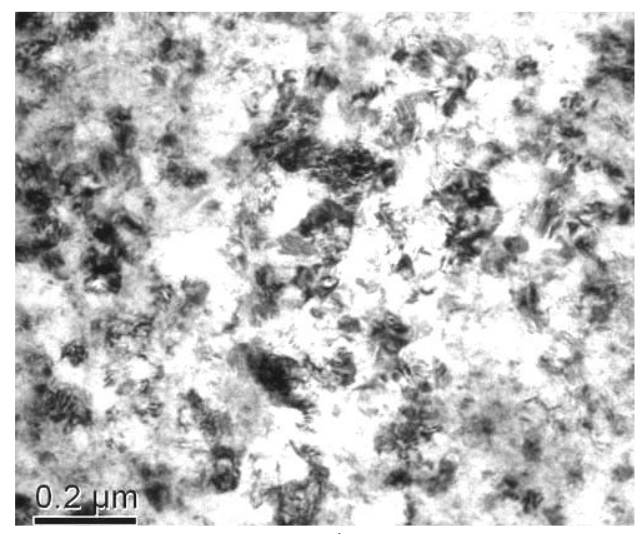

a)

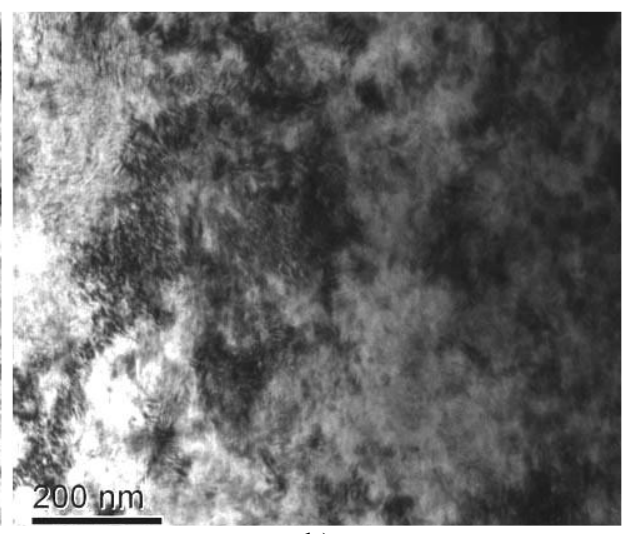

b)

Fig. 1. Electron microscopic images of the surface of a sample of aluminum-copper alloy after (a) and before (b) irradiation in an ultrasonic field for $10 \mathrm{~min}$.

The observed oxidation of the alloy can be explained by the action of radical particles (various forms of active oxygen), as well as the destruction of the initial oxide layer by cavitation jets [13-16]. The morphology of the surface formed as a result of processing also indicates that the formation of the oxide layer includes the processes of depassivationreoxidation.

According to $\mathrm{x}$-ray phase analysis, the resulting oxidation products are bayerite $\mathrm{Al}(\cdot \mathrm{H})$ 3 ; at the same time, as the dose load increases, additional boehmite lines $\mathrm{Al} \cdot(\cdot \mathrm{H})$ appear on X-ray diffraction patterns, this indicates the gradual development of dehydration processes under ultrasound exposure.

A detailed study of the structure of the oxidized layer was carried out using the electron microprobe method, which allows simultaneous recording of the relative contents of aluminum, oxygen, and copper at various points on the irradiated surface. From the profiles of the spatial distribution of elements along the scanning line by the electron beam, it 
follows that the bound oxygen is uniformly distributed over the surface of the sample subjected to ultrasonic irradiation, with the exception of areas corresponding to inclusions of the $\mathrm{A} 12 \mathrm{Cu}$ intermetallic compound. Near inclusions, oxidation proceeds much more efficiently, as a result of which an oxide layer of greater thickness is formed. The observed intensification of the process of aluminum oxidation can be explained by the effective reduction of the molecular oxygen process on copper-enriched inclusions under the influence of an ultrasonic field [17-20].

Light spots, correspond to areas enriched with copper, dark spots - elements of surface morphology (dimples); the line in the image corresponds to the trajectory of the electron beam when obtaining surface concentration profiles.

Sonochemical anodization is completed after the formation of a sufficiently thick and dense oxide layer encapsulating intermetallic inclusions. The latter circumstance should contribute to increasing the corrosion resistance of the alloy, since it is these inclusions that are the points of development of pitting corrosion in depassivating media. Electrochemical measurements indicate that the barrier layer created as a result of sonochemical treatment provides a sharp increase in the corrosion stability of an aluminum-copper alloy.

\section{Conclusion}

Thus, the ultrasonic treatment of an aluminum-copper alloy in an aqueous medium lead to the formation of a surface protective film, the process of its growth being highly selective and adaptive with negative feedback.

During ultrasonic treatment, the surface layer of aluminum alloy 1201 undergoes significant changes. A nanocrystalline structure with a grain size of less than 200 microns is formed in it. Metallographically, the nanocrystalline layer is detected as a strongly etched region, the thickness of which is determined by the parameters of ultrasonic treatment and is approximately $20-30 \mu \mathrm{m}$.

Implantation of aluminum alloy 1201 ions by cathode of $\mathrm{Cu}-\mathrm{Pb}$ system without ultrasonic treatment and after it showed that the depth of penetration of copper and lead ions during irradiation of the target without preliminary treatment is 320-380 nm. Ultrasonic treatment of the surface of the target from alloy 1201 before implantation leads to a decrease in the depth of penetration of ions to $160-180 \mathrm{~nm}$ and the appearance of amorphization in the implanted layer.

This work was carried out as part of the state task of the Ministry of Education and Science of Russia "The influence of magnetic fields and ion implantation on the structure, chemical composition and properties of titanium, aluminum alloys and elementary semiconductors".

\section{References}

1. A.V. Byeli, Wear, 203, 596-607 (1997)

2. P.J. Wilbur, Surface and Coatings Technology, 83 (1-3), 250-256 (1996)

3. A.N. Didenko, Yu.P. Sharkeyev, E.V. Kozlov, A.I. Ryabchikov, NTL Publishing House, 328 (2004)

4. Yu.I. Lopukhov, M.A. Lakhina, Engineering - from theory to practice, 4 (41), 51-59 (2015)

5. A.V. Kirichek, D.L. Soloviev, A.G. Lazutkin, Mechanical Engineering, 288 (2004)

6. M.A. Balter. Engineering, 184 (1978)

7. A.G. Tkachev, Publishing house of Tamb. state tech. University, 164 (2009)

8. O.V. Abramov, I.G. Khorbenko, S. Shvegla, Mechanical Engineering, 280 (1984)

9. V.Ye. Panin, Physical Mesomechanics, 9(2), (2006) 
10. V.E. Panin, Physical Mesomechanics, 8 (2005)

11. V.A. Klimenov, Physical Mesomechanics, 7 (2004)

12. O.V. Taratynov, V.V. Poroshin, V.V. Kharchenko, High technology in mechanical engineering, 10, 13-19 (2012)

13. D.D. Papshev, Finishing and hardening by surface plastic deformation, Mechanical Engineering, 152 (1978)

14. A.G. Suslov, R.V. Gurov, E.S. Tishevskikh, Hardening technologies and coatings, 9, 20-22 (2008)

15. V.P. Fedorov, M.N. Nagorkin, I.L. Pyrikov, Bulletin of the Bryansk State Technical University, 1, 22-31 (2011)

16. G.G. Poznyak, V.V. Kopylov, V.A. Rogov, Modern methods of designing nanotechnological processes in mechanical engineering, RUDN, 152 (2008)

17. V.V. Popova, Surface plastic deformation and physicochemical treatment, Rubtsovsky Industrial Institute, 98 (2013)

18. A. I. Lotkov, Letters in ZhTF, 31 (21), 24-29 (2005)

19. A.D. Pogrebnyak, Questions of atomic science and technology, 29(6) 571-577 (2008)

20. Yu.F. Ivanov, Fundamental problems of modern materials science. 5 (1), 47-52 (2008) 